\title{
New practice guidelines for perioperative beta blockade from the United States and Europe: incremental progress or a necessary evil?
}

\author{
Martin J. London, MD
}

Published online: 4 February 2010

(C) Canadian Anesthesiologists' Society 2010

In the latter half of 2009, practice guidelines were published that either dealt exclusively with perioperative beta blockade (as a focused update from the American College of Cardiology Foundation/American Heart Association [ACCF/AHA]) or included beta blockade within new comprehensive perioperative guidelines (from a task force of the European Society of Cardiology [ESC]) (Table 1). ${ }^{1-3}$ A major impetus was the impact of the controversial PeriOperative Ischemic Evaluation Trial (POISE) that originated in Canada and was coordinated from Canada. ${ }^{4}$ The results of this trial have been well publicized in a variety of opinion pieces. The study involved over 8,000 high-risk patients, more than $80 \%$ of whom had known coronary, peripheral arterial, or cerebrovascular disease and $40 \%$ of whom had undergone vascular surgery. In all cases, beta blockade began immediately prior to surgery with orally administered sustained release metoprolol (succinate) that continued for 30 days. $^{5-9}$ The study's primary findings posed substantial challenges to each guidelines committee. In particular, the efficacy in reducing cardiac ischemic complications (primarily asymptomatic troponin release, but also the need for revascularization and new atrial fibrillation) was counterbalanced most convincingly by an increased rate of stroke (primarily disabling) and, to a less striking statistical degree, death (primarily from

The author is not supported by, nor maintains any financial interest in, any commercial activity that may be associated with the topic of this article.

M. J. London, MD ( $\square)$

Department of Anesthesia and Perioperative Care, University of California, Veterans Affairs Medical Center, Anesthesia Service (129), 4150 Clement St, San Francisco, CA 94121, USA

e-mail: londonm@anesthesia.ucsf.edu sepsis), with "clinically significant" hypotension a strong attributable risk factor in both.

Considerable pressure was on the ACCF/AHA committee in the United States, where Class 1 recommendations form the basis for performance measures affecting hospitals and clinicians economically, either favourably or adversely, depending on their compliance to the guidelines. ${ }^{10}$ On either side of the Atlantic (hopefully, the Pacific as well), the ethical stakes were even higher, given the millions of at risk and aging patients undergoing a wide array of elective surgical procedures that either prolong or improve quality of life. Thus, proper recommendations are essential. In the late 1990s to the early 2000s, initial widespread enthusiasm for beta blockade as a "magic bullet" therapy led to beta blocker fatigue by many perioperative clinicians following publication of the neutral DIPOM and MAVS trials and the blockbuster POISE study.

Since its publication, the nearly universal (and often emotionally heated) consensus appears that the POISE protocol was indeed somewhat dangerous given the generous dose of metoprolol that was acutely administered four hours before surgery (to ensure an adequate level of physiologic beta blockade necessary in treating the thousands of diverse patients in the study). Yet, it does bear emphasizing that this criticism borders on "historical revisionism" given the overzealous promotion of perioperative beta blockade at the time the POISE protocol was first drafted to large groups of patients based on weak data or inappropriate extrapolation from highly selected patient subgroups. Regardless, the US guidelines group likely recognized a priori that it would be impossible to attempt to scrutinize the beneficial actions of beta blockade in the POISE study with sufficient precision to allow widespread implementation in a logistically feasible and safe manner. This problem was compounded by a lack of other publications from the POISE group on key methods, 
Table 1 Comparison of ACCF/AHA and ESC recommendations and levels of evidence for perioperative beta blockade

\begin{tabular}{|c|c|c|c|}
\hline & Class I Recommendation & Class IIa and Class IIb Recommendation & Class III Recommendation \\
\hline ACCF/AHA & $\begin{array}{l}\text { 1) Continuation in patients using } \\
\text { beta blockers for other ACCF/ } \\
\text { AHA Class I guideline } \\
\text { indications (Level C) }\end{array}$ & $\begin{array}{l}\text { 1(a) Undergoing vascular surgery at high } \\
\text { risk based on CAD or ischemia on } \\
\text { preoperative testing (Level B) } \\
\text { 2(a) Undergoing vascular surgery at high } \\
\text { cardiac risk based on more than one } \\
\text { clinical risk factor† (Level C) } \\
\text { 3(a) Undergoing intermediate risk } \\
\text { surgery§ with known IHD or high } \\
\text { cardiac risk with more than one clinical } \\
\text { risk factor(Level B) } \\
\text { 1(b) Undergoing intermediate risk or } \\
\text { vascular surgery with a single clinical } \\
\text { risk factor in the absence of IHD } \\
\text { 2(b) Undergoing vascular surgery with no } \\
\text { clinical risk factors, not currently } \\
\text { taking beta blockers (Level B) }\end{array}$ & $\begin{array}{l}\text { 1) Presence of absolute contraindications } \\
\text { to beta blockade (Level C) } \\
\text { 2) Use of high-dose beta blockers in the } \\
\text { absence of dose titration (Level B) }\end{array}$ \\
\hline ESC & $\begin{array}{l}\text { 1) Known IHD or myocardial } \\
\text { ischemia on preoperative stress } \\
\text { testing (Level B) } \\
\text { 2) Patients undergoing high-risk } \\
\text { surgery (Level B)* } \\
\text { 3) Continuation for patients using } \\
\text { beta blockers for CAD, } \\
\text { arrhythmias, or HTN (Level C) }\end{array}$ & $\begin{array}{l}\text { 1(a) Undergoing intermediate risk } \\
\text { surgery** (Level B) } \\
\text { 2(a) Continuation in chronic heart failure } \\
\text { patients with systolic dysfunction } \\
\text { (Level C) } \\
\text { 1(b) Undergoing low-risk surgery } \dagger \dagger \text { with } \\
\text { risk factors }\end{array}$ & $\begin{array}{l}\text { 1) Use of high-dose beta blockade without } \\
\text { dose titration (Level A) } \\
\text { 2) Undergoing low-risk surgery without } \\
\text { risk factors (Level B) }\end{array}$ \\
\hline
\end{tabular}

ACCF/AHA = American College of Cardiology Foundation/American Heart Association; ESC = European Society of Cardiology; $\mathrm{IHD}=$ ischemic heart disease; $\mathrm{CAD}=$ coronary artery disease; $\mathrm{HTN}=$ hypertension

* ESC high-risk surgery $=$ aortic and major vascular surgery or peripheral vascular surgery

$\dagger$ ACCF/AHA clinical risk factors $=$ history of ischemic heart disease, compensated heart failure, cerebrovascular disease, diabetes mellitus, and renal insufficiency (preoperative serum creatinine $>2 \mathrm{mg} \cdot \mathrm{dL}^{-1}$ )

$\S \mathrm{ACCF} / \mathrm{AHA}$ Intermediate risk surgery $=$ cardiac risk $1-5 \%=$ intraperitoneal and intrathoracic surgery, carotid endarterectomy, head and neck surgery, orthopedic surgery, prostate surgery

** ESC Intermediate risk surgery $=$ cardiac risk $1-5 \%=$ abdominal, carotid, peripheral arterial angioplasty; endovascular aneurysm repair; head and neck surgery; major neurological/orthopedic (hip and spine) surgery; transplant (lung, liver renal); and major urologic surgery

$\dagger \dagger$ ESC Low risk surgery $=<1 \%$ cardiac risk = breast, dental, endocrine, eye, gynecologic, reconstructive, minor orthopedic (knee), and minor urologic surgery

- ESC risk factors = ischemic heart disease, heart failure, cerebrovascular disease, diabetes mellitus requiring insulin, renal dysfunction/ hemodialysis, and age

Adapted with permission from Table 2 Fleischmann et al. 2009 and Poldermans et al. 2009 (Table page 16)

issues, or longer term outcome data other than that reported in the initial manuscript. In Europe, with its polyglot of countries and healthcare systems, such pressures were likely less of a factor, as their guidelines are generally considerably more liberal.

The POISE study's impact on the guidelines is strikingly similar to the influence the COMMIT trial had on ACCF/ AHA. The COMMIT trial involved over 45,000 patients and investigated the widely used practice of early (on hospital arrival) "aggressive" therapy with intravenous metoprolol in medical patients sustaining myocardial infarction. ${ }^{13}$ In that study, the efficacy of ventricular fibrillation and the reductions in the occurrence of reinfarction were counterbalanced by an increase in cardiogenic shock (safety). This study, like POISE, had obvious limitations, most notably that it was conducted entirely in China. However, it directly influenced the new ACCF/AHA Guidelines and Performance Measures causing the prior recommendation for beta blocker prescription during hospital stay to be eliminated and limiting it to prescription at time of discharge, in the absence of a host of relative or absolute contraindications. ${ }^{14}$ Thus, the response of the ACCF/AHA group appears to be entirely appropriate, and use of a high-dose protocol administered acutely in the absence of dose titration is accorded a Class III recommendation.

Regarding existing or new Class I indications, several publications from the European group, Poldermans et al., strongly suggest that use of a low dose, slowly titrated to heart rate protocol with bisoprolol (a highly beta 1-selective agent with once daily dosing) is efficacious in a wide 
variety of surgical and patient risk strata. ${ }^{15-17}$ Nevertheless, it was likely apparent to the ACCF/AHA group that there is insufficient evidence to date for implementation of this potentially expensive and logistically complex process in the United States with its "diverse" health care system (complicated by the lack of substantial data from studies conducted in the United States). In all probability, this factor accounts for the lack of any new Class 1 recommendations. Indeed, the preceding Class I recommendation, i.e., to institute beta blockade in patients undergoing vascular surgery who have documented ischemia on preoperative testing, was "downgraded" to Class II with no formal explanation. Thus, the sole remaining Class I recommendation is to continue beta blockade in patients based on indications of other Class I ACCF/AHA guidelines. This recommendation is slightly reworded from the earlier guideline in which the terminology, "to treat angina, symptomatic arrhythmias, hypertension" was specifically highlighted. ${ }^{18}$ The revised wording may be related to changing or absent Class I medical recommendations. In particular, chronic beta blocker therapy for "uncomplicated" hypertension (e.g., in the absence of known or suspected $\mathrm{CAD}$ ) has come under criticism recently given inferior longterm outcomes relative to non-beta blocker regimens. Elevated afterload resulting from unfavorable alterations in peripheral pulse wave propagation and reflection present with atenolol despite similar brachial artery pressures (relative to amlodipine) is considered a key pathophysiologic factor. ${ }^{19,20}$

The primary focus of the existing (and now only) ACCF/AHA Class I indication relates to the variably documented hazards of discontinuing a beta blocker perioperatively. In the literature, the risk ranges from no danger to overwhelmingly dangerous depending on the patient cohort, the indication for their chronic use, and the study design used for analysis. ${ }^{21}$ Although there is now suspicion that continuation of beta blockade in the anemic patient may be detrimental, current data are insufficient to take action, and the current Class I recommendations are prudent $_{\text {practice. }}{ }^{22}$ Conversely, there is also evidence that its discontinuation in the anemic patient may be associated with an increase in perioperative medical infarction (PMI). ${ }^{23}$ Despite controversy regarding continuation of angiotensin converting enzyme inhibitors, this topic receives little consideration despite the high prevalence of concurrent chronic use of these medications. ${ }^{24,25}$

The most controversial clinical issue in this arena is clearly the new institution of beta blockade for prophylactic purposes in previously naïve patients, which remains in the Class II category (either a or b). There is well publicized criticism of the ACCF/AHA Guidelines process that details an excessive reliance on Class II recommendations due to a lack of high quality available evidence and supports the clinical observation that Class II recommendations are quite weak (despite grouping into the IIa or IIb subcategories or the more cryptic estimates of certainty of treatment effect levels $\mathrm{A}, \mathrm{B}$, or $\mathrm{C}$ that are also applied). ${ }^{26,27}$ With many institutions placing necessary emphasis (e.g., for reimbursement) on Class 1 recommendations in the face of constrained institutional resources, Class II programs are considerably less likely to be implemented or performed properly.

The ACCF/AHA Guidelines rely almost exclusively on the revised cardiac risk index (RCRI) predictors as the primary criteria for instituting beta blocker therapy. ${ }^{28}$ The RCRI has been criticized for its limitations, most notably a lack of discriminative ability in patients undergoing open abdominal aortic aneurysm repair in the original cohort. Nonetheless, it appears to be robust in its ability to predict major adverse outcome (particularly with modification in terms of other variables, such as age or aneurysm size and the extremes of the cumulative number of predictor variables); it is easy to use and involves no expense. ${ }^{29-32}$ Although there are a host of other sensitive and more specific predictors, primarily new biomarkers, such as the naturetic peptides or inflammatory reactive proteins, these involve considerable additional expense when applied to many patients. ${ }^{33}$ The committee discounts the efficacy of "minor predictors", including the Framingham long-term cardiac risk variables used in the original atenolol study by Mangano et al., thus reducing the pool of eligible candidates. $^{34}$ It remains to be seen if protocols using these predictors, some of which have been disseminated on the internet, can provide sufficient evidence of efficacy for future use in these guidelines.

The primary impact of the POISE study on these guidelines is clearly a heightened sensitivity to the hazards of "aggressive" beta blockade in previously naïve patients. Thus, both guideline groups emphasize a considerably more conservative regimen with regard to initial dosing, dose escalation, and the period of time over which therapy is initiated prior to surgery. This approach to the pharmacokinetics and dynamics of beta blockade is based on a limited number of perioperative studies by the Poldermans group in which institution of low doses of bisoprolol within a window of 30 days to 1 week prior to surgery is promoted (all of which attest to its apparent safety). ${ }^{15-17}$ The ESC group specifically recommends starting doses of either $2.5 \mathrm{mg}$ of bisoprolol or $50 \mathrm{mg}$ of metoprolol succinate, while the ACCF/AHA group makes no specific drug recommendation (perhaps recognizing the popularity of atenolol or the cost-effectiveness of metoprolol tartrate). The ESC group is more explicit in its recommendation to titrate the dose of either drug to maintain a resting heart rate of $60-70$ beats $\cdot \min ^{-1}$, while the ACCF/AHA group has liberalized its earlier recommendations of 60-65 
beats $\cdot \min ^{-1}$ to $60-80$ beats $\cdot \min ^{-1}$. While the upper range may facilitate development of ischemia in a percentage of patients, recognizing that the cardiac index is dependent on heart rate once stroke volume has reached a maximum as occurs in severe anemia, adds a margin of safety appropriate for the perioperative period. ${ }^{35}$ The medical literature on which these recommendations rest is based in large part on the long-term outcome of patients with known CAD or hypertension, suggesting better survival with heart rates in the $60-70$ beats $\cdot \min ^{-1}$ range but also reporting evidence of adverse survival with lower ranges. ${ }^{36-39}$ These controversies emphasize the problems of applying findings from a patient group in one context, i.e., long-term survival in medical patients, to the more dynamic and shorter term perioperative setting.

The association of heart rate control with beta blockade with perioperative outcome remains controversial. Conflicting meta-analyses have been reported that attempted to tease out the limited hemodynamic data presented in the major randomized controlled trials. Beattie et al. and Biccard et al. reached differing conclusions when analyzing the major randomized controlled trials on the topic of perioperative cardioprotection, but neither included data from POISE. ${ }^{40,41}$ The more sophisticated analysis of Beattie et al. was supportive of an association of heart rate control with the reduction in PMI, suggesting less effect with the shorter acting metoprolol tartrate preparation and an additive effect in patients on calcium channel blockers. Both studies note an increase in drug associated adverse events with better heart rate control. In a wider reaching analysis criticized for inclusion of peripheral studies, e.g., the impact of beta blockers solely on hemodynamics during tracheal intubation, Bangalore et al. reported that a mean heart rate of $\leq 75$ beats. $\min ^{-1}$ at the end of the study was associated with a reduction in non-fatal myocardial infarction. $^{42}$

Processes of health care delivery must be taken into account and may differ substantially between countries. Given the controversy regarding the optimal means for instituting beta blockade, several smaller scale implementation studies have reported examining the use of dose titration $v s$ fixed dosing, which is accorded substantial attention in the guidelines. Surprisingly, neither guidelines group reported on a recent multi-centre randomized trial of 400 patients (approximately half of whom had preoperative dobutamine stress testing) performed in Australia where usual (non titrated) care was compared with an algorithmbased nurse-led protocol in which low-dose bisoprolol was started at least seven days prior to surgery and titrated to achieve a heart rate of 60 beats $\cdot \min ^{-1} \cdot{ }^{43}$ Cardiac ischemic events were noted in approximately $6 \%$ of both groups, with patients in the titrated group having a higher incidence of hypotension and bradycardia. This study provides timely data on prospectively tracked ischemic outcomes with all occurring on or before day four (excluding one at day 21).

Ultimately, there is little argument that beta blockers, calcium channel blockers, statins, alpha2 agonists, angiotensin receptor antagonists, aspirin, nitrates, and other agents exert clinically relevant cardioprotective effects when administered to the proper patient, in a particular milieu of physiologic conditions, and in the proper dosage for the optimal period of time; conversely, they are potentially dangerous when not administered accordingly. It is also clear that a host of other perioperative clinical interventions, e.g., epidural anesthesia/analgesia, other forms of pain management, thermal management strategies, can be significant covariates in the ultimate equation of perioperative morbidity and mortality. Other pharmacologic means of manipulating heart rate are on the horizon of perioperative clinical research, including possible use of the I(f) channel inhibitor, ivabradine, a selective bradycardic agent with no negative inotropic effects. ${ }^{44,45}$ It is also apparent that myocardial damage is a truly microscopic process that is likely ongoing in high risk patients on a daily basis in response to different stresses. ${ }^{46}$ With increasingly sensitive troponin assays being marketed, this damage will be seen on a daily basis, and, at present, it may be impossible to determine the cause, the preventative, and the long-term consequences. A recent report that low levels of troponin $\mathrm{T}$ have a long-term correlation with heart failure and cardiovascular death, but not myocardial infarction, fuels this new debate. ${ }^{47}$ The perioperative debate on the significance of silent myocardial ischemia as either a direct casual link or indirect bystander to an otherwise damaged heart (ongoing since the mid 1980s) has been heightened by recent large scale cardiology data demonstrating additive risk for even very short ischemic episodes following hospitalization for acute coronary syndromes. Other data suggests treatment may be beneficial in select patient groups. ${ }^{48-52}$ Yet, provocative testing that induces ischemia remains a routine part of cardiology practice, and ischemic preconditioning and now postconditioning are recognized forms of cardioprotection. Connecting the dots regarding diagnosis, detection, and appropriate treatment remains unfeasible conceptually and practically.

It can be argued that a stronger focus on the basic physiology, pharmacology, and epidemiology of the perioperative period is appropriate. Thus, much of the current controversy in this area regarding the role of blood pressure, anemia, and cerebral circulatory physiology in response to specific pharmacologic interventions, e.g., beta blockade, brought to the forefront by the POISE study, would perhaps have been predefined. ${ }^{53-55}$ Also, a stronger focus would guide the groups to relevant questions, such as: a) What are the parameters of physiologic beta blockade among various 
types of patients, including age, gender, race, and pharmacogenetics, and how are they influenced by concurrent physiologic processes, e.g., aging, anemia, transfusion, altered loading conditions? Although there is a rich background of literature, only passing consideration has been given to this topic by either group..$^{22,56-60}$ b) What are the pharmacokinetics and dynamics of each beta blocker, and how long is therapy required to institute or terminate their effects? Recent information blurs the monolithic class effect approach regarding efficacy between beta selective and non-selective agents. For example, ancillary "pleiotropic effects" and anti-oxidant and anti-inflammatory effects of carvedilol, a non-selective beta blocker, suggest it might be a better choice than bisoprolol, a hypothesis worth testing. ${ }^{61-64} \mathrm{c}$ ) What is the primary risk period for perioperative morbidity, and, in fact, are 30 days of therapy indicated? As noted above, there appears to be little evidence to support this duration of therapy. ${ }^{43}$ Admittedly, such a multi-parameter approach is exceedingly complex, but how else can these complex questions be answered? The current process appears to be limited increasingly to the "necessary evil" of winnowing out the few effective Class I practices from the dangerous Class III practices, leaving the majority of real world clinical controversy in the nebulous Class II category. Although it is possible that another large scale trial similar to POISE will be conducted, ironically it is unlikely, perhaps for the opposite reasons that made the POISE study so difficult; stroke or even death caused by a beta blocker will be the major fear, rather than a strong bias towards the preordained efficacy of beta blockers. In the 21 st century, it is imperative that our solutions keep pace with our rapidly evolving computerized learning and working environment. Unless absolutely indicated, let's not settle for a $20^{\text {th }}$ century necessary evil.

\section{Nouvelles directives de pratique pour l'administration périopératoire de bêta-bloquants aux Etats-Unis et en Europe: progrès marginal ou mal nécessaire?}

Durant la seconde partie de 2009, des directives de pratique ont été publiées, lesquelles traitaient soit exclusivement du bêta-blocage périopératoire (comme la mise à jour ciblée publiée par l'American College of Cardiology Foundation / American Heart Association [ACCF/AHA]), soit incluaient le bêta-blocage dans de nouvelles directives périopératoires globales (comme dans le cas du groupe de travail de la Société européenne de cardiologie [ESC]) (Tableau 1). ${ }^{1-3}$ L'étude POISE (PeriOperative Ischemic Evaluation Trial), qui a vu le jour au Canada et a été coordonnée depuis le Canada, a eu un impact significatif et a donné un élan majeur à la recherche dans ce domaine. ${ }^{4}$ Les résultats de cette étude ont été rendus publics dans toutes sortes d'articles d'opinion. L'étude a porté sur plus de 8000 patients à haut risque, dont plus de $80 \%$ souffraient de maladies coronarienne, artérielle périphérique ou vasculaire cérébrale connues, et $40 \%$ avaient déjà subi une chirurgie vasculaire. Dans tous les cas, le bêta-blocage a été débuté immédiatement avant la chirurgie à l'aide de (succinate de) métoprolol à libération prolongée administré oralement et maintenu pendant 30 jours. ${ }^{5-9}$ Les résultats primaires de l'étude ont posé d'importants défis à chaque comité de rédaction de directives. Plus particulièrement, l'efficacité du traitement dans la réduction des complications ischémiques cardiaques (principalement la libération asymptomatique de troponine, mais également le besoin de revascularisation et une nouvelle fibrillation auriculaire) a été contrebalancée de façon indubitable par un taux accru d'accidents vasculaires cérébraux (principalement invalidants) et, de façon moins frappante statistiquement parlant, de décès (principalement liés à une sepsie). Une hypotension « significative d'un point de vue clinique »a constitué un facteur de risque prononcé dans les deux cas.

Le comité de l'ACCF/AHA a subi des pressions considérables aux États-Unis, où les recommandations de classe I constituent le fondement des mesures de performance affectant économiquement—de façon positive ou négative-les hôpitaux et les cliniciens selon leur respect des directives. ${ }^{10}$ De part et d'autre de l'Atlantique (et probablement aussi du Pacifique), les enjeux éthiques jouaient un rôle encore plus important étant donné les millions de personnes à risque et de patients âgés subissant toutes sortes d'interventions chirurgicales non urgentes dans le but de prolonger ou d'améliorer la qualité de leur vie. Dès lors, il est essentiel de disposer de recommandations adaptées. Entre la fin des années 1990 et le début des années 2000, l'enthousiasme initial dont le bêta-blocage, alors décrit comme un traitement «miracle », a fait l'objet est retombé chez plusieurs cliniciens périopératoires à la suite de la publication des études neutres DIPOM et MAVS, puis de l'étude vedette POISE.

Depuis la publication de cette dernière, le consensus quasi unanime (et souvent chargé émotionnellement) semble être que le protocole POISE était effectivement un tant soit peu dangereux en raison de la dose généreuse de métoprolol administrée en phase aiguë, quatre heures avant la chirurgie. Cette posologie avait pour objectif de garantir un niveau suffisant de bêta-blocage physiologique, 
Tableau 1 Comparaison des recommandations ACCF/AHA et ESC et niveaux de données probantes pour le bêta-blocage périopératoire

\begin{tabular}{|c|c|c|c|}
\hline & Recommandation de classe I & Recommandation de classe IIa et IIb & Recommandation de classe III \\
\hline ACCF/AHA & $\begin{array}{l}\text { 1) Maintien chez les patients à } \\
\text { l'aide de bêta-bloquants pour } \\
\text { d'autres indications des } \\
\text { directives de classe I de } \\
\text { l'ACCF/AHA (niveau C) }\end{array}$ & $\begin{array}{l}\text { 1(a) Subissant une chirurgie vasculaire à } \\
\text { haut risque selon une maladie } \\
\text { coronarienne ou de l'ischémie lors des } \\
\text { tests préopératoires (niveau B) } \\
\text { 2(a) Subissant une chirurgie vasculaire à } \\
\text { haut risque cardiaque sur la base de } \\
\text { plus qu'un facteur de risque cardiaque† } \\
\text { (niveau C) } \\
\text { 3(a) Subissant une chirurgie de risque } \\
\text { modéré§ avec cardiomyopathie } \\
\text { ischémique connue ou risque cardiaque } \\
\text { élevé avec plus qu'un facteur de risque } \\
\text { clinique (niveau B) } \\
\text { 1(b) Subissant une chirurgie à risque } \\
\text { modéré ou vasculaire avec un seul } \\
\text { facteur de risque clinique en l'absence } \\
\text { de cardiomyopathie ischémique } \\
\text { 2(b) Subissant une chirurgie vasculaire } \\
\text { sans facteur de risque clinique et ne } \\
\text { prenant pas de bêta-bloquants } \\
\text { actuellement (niveau B) }\end{array}$ & $\begin{array}{l}\text { 1) Présence de contre-indications } \\
\text { absolues au bêta-blocage (niveau C) } \\
\text { 2) Utilisation de bêta-bloquants à forte } \\
\text { dose en l'absence d'ajustement (niveau } \\
\text { B) }\end{array}$ \\
\hline ESC & $\begin{array}{l}\text { 1) Cardiomyopathie ischémique ou } \\
\text { ischémie myocardique connue } \\
\text { lors des tests de stress } \\
\text { préopératoires (niveau B) } \\
\text { 2) Patients subissant une chirurgie } \\
\text { à risque élevé (niveau B)* } \\
\text { 3) Maintien chez les patients } \\
\text { utilisant des bêta-bloquants pour } \\
\text { maladie coronarienne, arythmie, } \\
\text { ou hypertension (niveau C) }\end{array}$ & $\begin{array}{l}\text { 1(a) Subissant une chirurgie à risque } \\
\text { modéré } * * \text { (niveau B) } \\
\text { 2(a) Maintien chez patients avec } \\
\text { insuffisance cardiaque chronique et } \\
\text { dysfonction systolique (niveau C) } \\
\text { 1(b) Subissant une chirurgie à faible } \\
\text { risque †† avec des facteurs de risque }\end{array}$ & $\begin{array}{l}\text { 1) Utilisation de bêta-blocage à forte dose } \\
\text { sans ajustement (niveau A) } \\
\text { 2) Subissant une chirurgie à faible risque } \\
\text { sans facteur de risque (niveau B) }\end{array}$ \\
\hline
\end{tabular}

$\mathrm{ACCF} / \mathrm{AHA}=$ American College of Cardiology Foundation/American Heart Association; ESC = Société européenne de cardiologie

* Chirurgie à risque élevé selon l'ESC = chirurgie aortique et vasculaire majeure ou chirurgie vasculaire périphérique

† Facteurs de risque cliniques de l'ACCF/AHA = antécédents de cardiomyopathie ischémique, insuffisance cardiaque compensée, maladie vasculaire cérébrale, diabète et insuffisance rénale (créatininémie préopératoire $>2 \mathrm{mg} \cdot \mathrm{dL}^{-1}$ )

$\S$ Chirurgie à risque modéré selon l'ACCF/AHA = risque cardiaque $1-5 \%=$ chirurgie intra-péritonéale et intra-thoracique, endartérectomie carotidienne, chirurgie de la tête et du cou, chirurgie orthopédique, chirurgie de la prostate

** Chirurgie à risque modéré selon l'ESC = risque cardiaque $1-5 \%=$ angioplastie abdominale, carotidienne, artérielle périphérique; chirurgie de réparation d'anévrisme endovasculaire; chirurgie de la tête et du cou; chirurgie neurologique/orthopédique (hanche et colonne) majeure; greffe (poumon, foie, rein); et chirurgie urologique majeure

$\dagger \dagger$ Chirurgie à faible risque selon l'ESC $=<1 \%$ risque cardiaque $=$ chirurgie mammaire, dentaire, endocrinienne, oculaire, gynécologique, de reconstruction, orthopédique mineure (genou) et urologique mineure

- Facteurs de risque selon l'ESC = cardiomyopathie ischémique, insuffisance cardiaque, maladie vasculaire cérébrale, diabète nécessitant de l'insuline, insuffisance rénale / hémodialyse, et âge

Adapté, avec permission, du tableau 2 de Fleischmann et coll. 2009 et Poldermans et coll. 2009 (tableau page 16)

nécessaire pour traiter les milliers de patients souffrant de conditions diverses dans l'étude. Il est néanmoins important de souligner que cette critique frise le « révisionnisme historique »: en effet, le bêta-blocage périopératoire faisait l'objet d'une promotion insensée à l'époque de l'élaboration du protocole POISE. En outre, il était utilisé auprès de larges groupes de patients sur la base de données peu probantes ou d'extrapolations inappropriées tirées de sous-groupes de patients très spécifiques. Indépendamment de ces facteurs, le groupe de rédaction des directives américaines a probablement réalisé a priori qu'il serait impossible d'analyser les bienfaits du bêta-blocage dans l'étude POISE avec suffisamment de précision pour permettre une mise en œuvre étendue qui soit à la fois faisable et sécuritaire. À ce problème s'est ajoutée l'absence d'autres publications du groupe POISE au sujet des données clés concernant la méthode, les problèmes rencontrés, ou les devenirs à plus long terme, 
soit des données différentes de celles rapportées dans le premier manuscrit. En Europe, en raison de la diversité de pays et de systèmes de santé, de telles pressions ont probablement joué un rôle moindre; par conséquent, leurs directives sont en règle générale considérablement plus libérales.

L'impact de l'étude POISE sur les directives ressemble de façon frappante à l'influence que l'étude COMMIT a eu sur l'ACCF/AHA. L'étude COMMIT portait sur plus de 45000 patients et a examiné la pratique très répandue de traitement 'agressif' précoce (à l'arrivée à l'hôpital) à base de métoprolol intraveineux chez les patients médicaux souffrant d'un infarctus du myocarde. ${ }^{13}$ Dans cette étude, l'efficacité de la fibrillation ventriculaire et les réductions dans la survenue d'un nouvel infarctus ont été neutralisées par une augmentation des chocs cardiogéniques (sécurité). Cette étude, tout comme l'étude POISE, comportait des limites évidentes, la plus importante étant qu'elle a été menée exclusivement en Chine. Cependant, l'étude COMMIT a eu une influence directe sur les Directives et sur les mesures de performance de l'ACCF/AHA, provoquant l'élimination de la recommandation précédente de prescription de bêta-bloquants pendant le séjour à l'hôpital et la limitant à une prescription au moment du congé, en l'absence d'une multitude de contre-indications relatives ou absolues. ${ }^{14}$ Par conséquent, la réaction du groupe de l'ACCF/AHA semble tout à fait adéquate, et l'utilisation d'un protocole à forte dose administré en phase aiguë en l'absence d'ajustement posologique reçoit une recommandation de classe III.

En ce qui concerne les indications de classe I existantes ou nouvelles, plusieurs publications de Poldermans et coll., le groupe européen, recommandent un protocole de faible dose de bisoprolol (un agent bêta 1 hautement sélectif à dosage quotidien unique) ajusté lentement en fonction de la fréquence cardiaque. Ce protocole serait efficace chez divers groupes de patients médicaux et chirurgicaux. ${ }^{15-17}$ Néanmoins, il est probable que le groupe de l'ACCF/AHA se soit rendu compte que les données probantes actuelles étaient insuffisantes pour justifier la mise en œuvre d'un tel processus aux États-Unis, étant donné son coût potentiellement élevé et sa complexité logistique aux États-Unis, un pays au système de soins de santé « diversifié » (le manque de données d'envergure tirées d'études menées aux États-Unis compliquant encore la donne). Selon toute probabilité, ce facteur est à l'origine de l'absence de nouvelles recommandations de classe I. En fait la recommandation de classe I précédente, soit la mise en place d'un bêta-blocage chez les patients subissant une chirurgie vasculaire et chez qui on avait observé une ischémie lors des tests préopératoires, a été reléguée à la classe II et ce, sans explication formelle. Dès lors, la seule recommandation de classe I restante est de maintenir le bêta-blocage chez les patients conformément aux indications d'autres directives de classe I de l'ACCF/AHA. Cette recommandation a été quelque peu reformulée comparativement à la directive précédente, dans laquelle la terminologie «pour traiter l'angine, les arythmies asymptomatiques, l'hypertension » était spécifiquement soulignée dans la version anglaise. ${ }^{18} \mathrm{La}$ révision du texte pourrait être liée à des changements au niveau des recommandations médicales de classe I, ou à l'absence de telles recommandations. En particulier, le traitement systématique aux bêta-bloquants pour l'hypertension «sans complication», par ex. en l'absence de maladie coronarienne connue ou soupçonnée, a été considérablement critiqué dans la littérature récente et a résulté en devenirs à long terme inférieurs. Cette réprobation est probablement liée à la surcharge élevée qu'on peut observer avec l'aténolol—mais non l'amlodipine-qui résulte d'altérations défavorables dans la propagation et la réflexion de l'onde de pression périphérique. ${ }^{19,20}$

L'indication de classe I de l'ACCF/AHA existante (désormais la seule) se concentre sur les dangers plus ou moins documentés d'une interruption périopératoire du traitement aux bêta-bloquants. Dans la littérature, le risque va de 'sans danger' à 'extrêmement dangereux' selon la cohorte de patients, l'indication pour leur utilisation systématique, et la méthodologie d'étude utilisée lors de l'analyse des données. ${ }^{21}$ Bien qu'il soit désormais pressenti que le maintien du bêta-blocage chez le patient anémique pourrait avoir des effets néfastes, les données actuelles ne suffisent pas pour se prononcer, et les recommandations actuelles de classe I appellent à la prudence. ${ }^{22}$ À l'inverse, il existe aussi des données probantes selon lesquelles l'interruption d'un tel traitement chez un patient anémique pourrait être associée à une augmentation des infarctus du myocarde périopératoires. ${ }^{23}$ Malgré la controverse qui entoure le maintien des inhibiteurs de l'enzyme de conversion de l'angiotensine, ce sujet n'est que peu traité en dépit de la prévalence importante d'utilisation simultanée de ces médicaments sur une base chronique. ${ }^{24,25}$

La question clinique la plus controversée dans ce domaine est clairement l'introduction du bêta-blocage à des fins prophylactiques chez des patients jusqu'alors naïs, une indication qui demeure dans la catégorie de classe II (a ou b). Des critiques bien connues du processus d'élaboration des Directives de l'ACCF/AHA détaillent la confiance excessive accordée aux recommandations de classe I en raison de l'absence de données probantes de qualité. En outre, ces critiques abondent dans le sens de l'observation clinique selon laquelle les recommandations de classe II sont plutôt faibles (malgré leur regroupement en sous-catégories IIa ou IIb, ainsi que les estimations quelque peu obscures de certitudes des niveaux d'effet du traitement en $\mathrm{A}, \mathrm{B}$ ou $\mathrm{C}$, également appliquées). ${ }^{26,27}$ Étant donné que plusieurs institutions 
insistent fortement (par ex. pour le remboursement) sur les recommandations de classe $I$ en raison des limites institutionnelles en matière de ressources, il est nettement moins probable que des programmes de classe II soient mis en place ou réalisés correctement.

Les Directives de l'ACCF/AHA s'appuient quasi exclusivement sur les prédicteurs de l'indice de risque cardiaque modifié comme critères principaux pour la mise en place d'un traitement de bêta-blocage. ${ }^{28}$ Cet indice a été critiqué pour ses limites, la plus importante étant l'absence de capacité discriminatoire chez les patients subissant une chirurgie ouverte de réparation d'anévrisme aortique abdominal dans la cohorte d'origine. Cependant, cet indice semble être à même de bien prédire les complications majeures (particulièrement lors de modifications d'autres variables, comme l'âge ou la taille de l'anévrisme et les extrêmes du nombre cumulé des variables de prédiction); enfin, il est facile à utiliser et ne coûte rien. ${ }^{29-32}$ Bien qu'il existe une multitude d'autres prédicteurs sensibles et plus spécifiques, principalement des nouveaux biomarqueurs tels que les peptides natriurétiques ou les protéines réactives inflammatoires, ces prédicteurs engendrent d'importants coûts supplémentaires lorsqu'ils sont appliqués à un nombre important de patients. ${ }^{33}$ Le comité ne tient pas compte de l'efficacité des «prédicteurs mineurs », notamment des variables de risque cardiaque à long terme de Framingham utilisées dans l'étude originale sur l'aténolol de Mangano et coll., ce qui réduit encore plus le bassin de candidats potentiels. ${ }^{34}$ Il reste à déterminer si les protocoles utilisant ces prédicteurs, dont certains ont été diffusés sur l'Internet, peuvent fournir suffisamment de données probantes quant à leur efficacité, afin d'être inclus dans ces directives à l'avenir.

L'impact principal de l'étude POISE sur ces directives se retrouve surtout dans une sensibilité accrue aux risques liés à un bêta-blocage «agressif » chez des patients précédemment naïs. Pour cette raison, les deux groupes de rédaction de directives recommandent un régime considérablement plus conservateur quant au dosage initial, à l'intensification des doses et au moment de début du traitement avant la chirurgie. Cette approche de la pharmacocinétique et de la dynamie du bêta-blocage se fonde sur un nombre restreint d'études périopératoires de Poldermans et coll., dans lesquelles l'introduction de faibles doses de bisoprolol est recommandée dans une fenêtre de 30 jours à une semaine avant la chirurgie (toutes les études font état de l'apparente innocuité du médicament). ${ }^{15-17}$ Le groupe de l'ESC recommande spécifiquement des doses initiales de $2,5 \mathrm{mg}$ de bisoprolol ou $50 \mathrm{mg}$ de succinate de métoprolol, alors que le groupe de l'ACCF/AHA n'émet pas de recommandation médicamenteuse spécifique (reconnaissant peut-être la popularité de l'aténolol ou la rentabilité du tartrate de métoprolol). Le groupe ESC est plus explicite dans sa recommandation de titrer la dose de l'un ou l'autre médicament afin de maintenir une fréquence cardiaque au repos de $60-70$ battements. $\min ^{-1}$, alors que le groupe de 1'ACCF/AHA a assoupli ses recommandations précédentes de 60-65 battements $\cdot \min ^{-1}$ à 60-80 battements $\cdot \min ^{-1}$. Alors que la plage supérieure de ces limites pourrait favoriser une ischémie chez un certain pourcentage des patients, le fait de reconnaître que l'index cardiaque dépend de la fréquence cardiaque une fois que le volume d'éjection a atteint un maximum, comme cela arrive dans les cas d'anémie grave, ajoute une marge de sécurité adéquate en période périopératoire. ${ }^{35}$ La littérature médicale sur laquelle ces recommandations sont basées se fonde en grande partie sur le devenir à long terme de patients souffrant de maladie coronarienne connue ou d'hypertension, et suggère un taux de survie plus élevé lorsque les fréquences cardiaques se situent dans la fenêtre des $60-70$ battements $\cdot \min ^{-1}$. Toutefois, cette littérature rapporte également une survie diminuée lorsque la fenêtre a des valeurs plus basses. ${ }^{36-39}$ Toutes ces controverses mettent en exergue les problèmes qui émergent lorsqu'on tente d'appliquer les résultats d'un groupe de patients dans un contexte donné, soit la survie à long terme de patients médicaux, au contexte périopératoire, lequel est à la fois plus dynamique et à plus court terme.

L'association entre le contrôle de la fréquence cardiaque par bêta-blocage et le devenir périopératoire demeure controversée. On a rapporté des méta-analyses contradictoires qui tentaient de patiemment démêler les données hémodynamiques limitées présentées dans les principales études randomisées contrôlées. Beattie et coll. et Biccard et coll. sont parvenus à des conclusions différentes lors de leurs analyses des études randomisées contrôlées principales portant sur la cardioprotection périopératoire, mais ni l'un ni l'autre n'ont inclus les données de l'étude POISE. ${ }^{40,41}$ L'analyse de Beattie et coll., plus raffinée, soutient qu'il existe une association entre le contrôle de la fréquence cardiaque et une réduction des infarctus du myocarde périopératoire, ce qui suggère un effet moindre lors de l'utilisation de la préparation à base du tartrate de métoprolol, à durée d'action plus courte, et un effet additif chez les patients traités avec des inhibiteurs calciques. Les deux études notent une augmentation des complications associées aux médicaments avec un meilleur contrôle de la fréquence cardiaque. Dans une analyse de plus grande envergure critiquée pour son inclusion d'études périphériques, comme par exemple l'impact des bêta-bloquants exclusivement sur l'hémodynamie pendant l'intubation trachéale, Bangalore et coll. rapportent qu'une fréquence cardiaque moyenne de $\leq 75$ battements $\cdot \min ^{-1}$ à la fin de l'étude était associée à une réduction des infarctus du myocarde non fatals. ${ }^{42}$ 
Il faut tenir compte des processus de prestation des soins de santé, qui peuvent être très différents d'un pays à l'autre. Étant donné la controverse entourant la meilleure façon de débuter le bêta-blocage, plusieurs études de mise en œuvre de petite envergure se sont concentrées sur la comparaison d'un ajustement des doses par rapport à un dosage fixe. En effet, l'ajustement occupe une place importante dans les directives. Il est étonnant qu'aucun des deux groupes d'élaboration des directives n'ait mentionné une étude randomisée multicentrique récente portant sur 400 patients (dont environ la moitié avait subi une épreuve de stress sous dobutamine avant l'opération) réalisée en Australie. Cette étude a comparé les soins habituels (pas d'ajustement) à un protocole basé sur un algorithme et réalisé par les infirmières dans lequel du bisoprolol à faible dose a été administré au moins sept jours avant la chirurgie et ajusté afin d'atteindre une fréquence cardiaque de 60 battements. $\min ^{-1}{ }^{43}$ Des événements ischémiques cardiaques ont été rapportés chez environ $6 \%$ des patients de chaque groupe, les patients du groupe d'ajustement manifestant une incidence plus élevée d'hypotension et de bradycardie. Cette étude présente des données opportunes sur les devenirs ischémiques suivis de façon prospective, ces événements survenant tous au jour quatre ou avant (à l'exception d'un patient, au jour 21).

En fin de compte, il n'est plus à prouver que les bêta-bloquants, les inhibiteurs calciques, les statines, les agonistes alpha-2, les antagonistes des récepteurs de l'angiotensine, l'aspirine, les nitrates et d'autres agents exercent des effets cardioprotecteurs pertinents d'un point de vue clinique lorsqu'ils sont administrés au bon patient, dans un milieu particulier de conditions physiologiques et dans un dosage adéquat pour une période de temps optimale. À l'inverse, ces agents sont tous potentiellement dangereux s'ils sont mal administrés. Il est également clair que plusieurs autres interventions cliniques périopératoires, par ex. l'anesthésie/analgésie péridurale, les autres formes de prise en charge de la douleur, les stratégies de prise en charge de la température, etc. peuvent constituer des co-variables importantes dont il faut tenir compte dans l'équation finale de la morbidité et de la mortalité périopératoires. Bientôt, d'autres méthodes pharmacologiques permettant de manipuler la fréquence cardiaque verront le jour dans la recherche clinique périopératoire. Par exemple, il est possible que l'inhibiteur du canal I(f), l'ivabradine, un agent bradycardisant sélectif sans effet inotrope négatif, soit utilisé. ${ }^{44,45} \mathrm{Il}$ est également évident que la lésion myocardique est un processus véritablement microscopique qui perdure probablement quotidiennement chez les patients à haut risque en réponse à différents stress. ${ }^{46}$ Avec la commercialisation de tests de troponine de plus en plus sensibles, ce genre de lésion sera observé quotidiennement. Toutefois, à l'heure actuelle, il demeure encore impossible de déterminer la cause, les mesures de prévention, ou les conséquences à long terme. Un compte-rendu récent, qui rapporte que des niveaux bas de troponine $\mathrm{T}$ ont une corrélation à long terme avec l'insuffisance cardiaque et le décès cardiovasculaire, mais pas avec l'infarctus du myocarde, alimente ce nouveau débat. ${ }^{47}$ Le débat sur le sens de l'ischémie myocardique silencieuse en périopératoire en tant que lien causal direct ou spectateur indirect d'un cœur autrement endommagé a commencé au milieu des années 1980. Ce débat a récemment atteint un sommet en raison de la publication de données cardiologiques d'envergure démontrant un risque additif même dans le cas d'épisodes très courts d'ischémie à la suite d'une hospitalisation pour des syndromes coronariens aigus. D'autres données suggèrent qu'un traitement pourrait être bénéfique à certains groupes de patients choisis. ${ }^{48-52}$ Et pourtant, les tests provocateurs induisant l'ischémie font encore partie de la pratique courante en cardiologie, et le pré-conditionnement ischémique-et maintenant le post-conditionnement - constituent des formes reconnues de cardioprotection. Toutefois il demeure impossible, tant sur le plan conceptuel que pratique, d'établir des liens quant au diagnostic, au dépistage et au traitement adéquat.

On peut avancer qu'une emphase plus soutenue sur la physiologie, la pharmacologie et l'épidémiologie de base de la période périopératoire serait appropriée. Ainsi, une grande partie de la controverse dans ce domaine concernant le rôle de la tension artérielle, de l'anémie et de la physiologie circulatoire cérébrale en réaction à des interventions pharmacologiques spécifiques, comme par exemple le bêta-blocage, et mise en exergue par l'étude POISE, aurait peut-être été prédéfinie. ${ }^{53-55}$ De plus, une direction plus claire aiderait les différents groupes à se concentrer sur des questions pertinentes telles que : a) Quels sont les paramètres du bêta-blocage physiologique chez différents types de patients, y compris l'âge, le sexe, la race et la pharmacogénétique, et comment sont-ils influencés par des processus physiologiques survenant simultanément, comme le vieillissement, l'anémie, la transfusion, des conditions de charge modifiées? Bien que la littérature dans le domaine soit abondante, une attention que très limitée n'a été accordée à ce sujet par l'un et l'autre groupe d'élaboration des directives. ${ }^{22,56-60}$ b) Quelles sont la pharmacocinétique et la dynamie de chaque bêta-bloquant, et quelle est la durée nécessaire de traitement pour qu'ils fassent effet-ou au contraire cessent de faire effet? Des données récentes brouillent l'approche monolithique de l'effet de classe quant à l'efficacité entre les agents bêta-sélectifs et non sélectifs. Par exemple, les «effets pléiotropiques » accessoires et les effets antioxydants et anti-inflammatoires du carvédilol, un bêta-bloquant non sélectif, laissent à penser que cet agent pourrait constituer une meilleure option que le bisoprolol. En tous les cas, l'hypothèse mérite d'être mise à 
l'épreuve. $\left.{ }^{61-64} \mathrm{c}\right)$ Quelle est la période de risque primaire de morbidité périopératoire et, en fait, est-ce que 30 jours de traitement sont indiqués? Tel que mentionné ci-dessus, il semble y avoir peu de données probantes soutenant une telle durée de traitement. ${ }^{43}$ Certes, une telle approche multi-paramétrique est extrêmement complexe, mais comment peut-on répondre à ces questions complexes autrement? Le processus utilisé actuellement semble être de plus en plus limité par le «mal nécessaire » d'écarter les quelques pratiques efficaces de la classe I des pratiques dangereuses de la classe III, ce qui laisse la vaste majorité des controverses cliniques réelles dans la catégorie quelque peu nébuleuse de la classe II. Bien qu'il soit possible qu'une autre étude d'envergure semblable à POISE soit réalisée, l'ironie veut que ce soit peu probable, peut-être pour les raisons opposées à celles qui ont rendu l'étude POISE si difficile. Dans une telle étude, la peur principale serait l'accident vasculaire cérébral, voire le décès causé par un bêta-bloquant plutôt qu'un important biais en faveur de l'efficacité présupposée des bêta-bloquants. Au $21^{\mathrm{e}}$ siècle, il est essentiel que nos solutions évoluent au même pas rapide que nos environnements informatisés de travail et d'apprentissage. À moins que cela ne soit absolument indiqué, ne nous contentons pas d'un mal nécessaire du $20^{\mathrm{e}}$ siècle.

Conflicts of interest None declared.

\section{References}

1. Fleischmann KE, Beckman JA, Buller CE, et al. 2009 ACCF/ AHA focused update on perioperative beta blockade: a report of the American College of Cardiology Foundation/American Heart Association Task Force on Practice Guidelines. Circulation 2009; 120: 2123-51.

2. Fleisher LA, Beckman JA, Brown KA, et al. 2009 ACCF/AHA focused update on perioperative beta blockade incorporated into the ACC/AHA 2007 guidelines on perioperative cardiovascular evaluation and care for noncardiac surgery: a report of the American College of Cardiology Foundation/American Heart Association Task Force on Practice Guidelines. Circulation 2009; 120: e169-276.

3. Poldermans D, Bax JJ, Boersma E, et al. Guidelines for preoperative cardiac risk assessment and perioperative cardiac management in non-cardiac surgery: The Task Force for Preoperative Cardiac Risk Assessment and Perioperative Cardiac Management in Non-cardiac Surgery of the European Society of Cardiology (ESC) and endorsed by the European Society of Anaesthesiology (ESA). Eur Heart J 2009; 30: 2769-812.

4. Devereaux PJ, Yang H, Yusuf S, et al. Effects of extended-release metoprolol succinate in patients undergoing non-cardiac surgery (POISE trial): a randomised controlled trial. Lancet 2008; 371: 1839-47.

5. Auerbach $A D$. Changing the practice of perioperative cardioprotection: perioperative $\{$ beta\}-blockers after POISE (PeriOperative ISchemic Evaluation). Circ Cardiovasc Qual Outcomes 2008; 1 : 58-61.
6. London MJ. Quo vadis, perioperative beta blockade? Are you "POISE'd" on the brink? Anesth Analg 2008; 106: 1025-30.

7. Sear JW, Giles JW, Howard-Alpe G, Foex P. Perioperative betablockade, 2008: what does POISE tell us, and was our earlier caution justified? Br J Anaesth 2008; 101: 135-8.

8. Yang H, Beattie WS. POISE results and perioperative betablockade. Can J Anesth 2008; 55: 727-34.

9. Chopra V, Plaisance B, Cavusoglu E, Flanders SA, Eagle KA. Perioperative beta-blockers for major noncardiac surgery: Primum Non Nocere. Am J Med 2009; 122: 222-9.

10. Spertus JA, Eagle KA, Krumholz HM, Mitchell KR, Normand SL; American College of Cardiology; American Heart Association Task Force on Performance Measures. American College of Cardiology and American Heart Association methodology for the selection and creation of performance measures for quantifying the quality of cardiovascular care. Circulation 2005; 111: 170312.

11. Ko DT, Hebert PR, Coffey CS, Sedrakyan A, Curtis JP, Krumholz $H M$. Beta-blocker therapy and symptoms of depression, fatigue, and sexual dysfunction. JAMA 2002; 288: 351-7.

12. London MJ. Beta blockers and alpha 2 agonists for cardioprotection. Best Pract Res Clin Anaesthesiol 2008; 22: 95-110.

13. Chen ZM, Pan HC, Chen YP, et al. Early intravenous then oral metoprolol in 45, 852 patients with acute myocardial infarction: randomised placebo-controlled trial. Lancet 2005; 366: 1622-32.

14. Krumholz HM, Anderson JL, Bachelder BL, et al. ACC/AHA 2008 performance measures for adults with ST-elevation and non-ST-elevation myocardial infarction: a report of the American College of Cardiology/American Heart Association Task Force on Performance Measures (Writing Committee to develop performance measures for ST-elevation and non-ST-elevation myocardial infarction): developed in collaboration with the American Academy of Family Physicians and the American College of Emergency Physicians: endorsed by the American Association of Cardiovascular and Pulmonary Rehabilitation, Society for Cardiovascular Angiography and Interventions, and Society of Hospital Medicine. Circulation 2008; 118: 2596-648.

15. Poldermans D, Boersma E, Bax JJ, et al. The effect of bisoprolol on perioperative mortality and myocardial infarction in high-risk patients undergoing vascular surgery. Dutch Echocardiographic Cardiac Risk Evaluation Applying Stress Echocardiography Study Group. N Engl J Med 1999; 341: 1789-94.

16. Poldermans D, Schouten $O$, Vidakovic $R$, et al. A clinical randomized trial to evaluate the safety of a noninvasive approach in high-risk patients undergoing major vascular surgery: the DECREASE-V Pilot Study. J Am Coll Cardiol 2007; 49: 1763-9.

17. Dunkelgrun M, Boersma E, Schouten O, et al. Bisoprolol and fluvastatin for the reduction of perioperative cardiac mortality and myocardial infarction in intermediate-risk patients undergoing noncardiovascular surgery: a randomized controlled trial (DECREASE-IV). Ann Surg 2009; 249: 921-6.

18. Fleisher LA, Beckman JA, Brown KA, et al. ACC/AHA 2007 Guidelines on Perioperative Cardiovascular Evaluation and Care for Noncardiac Surgery: Executive Summary: A Report of the American College of Cardiology/American Heart Association Task Force on Practice Guidelines (Writing Committee to Revise the 2002 Guidelines on Perioperative Cardiovascular Evaluation for Noncardiac Surgery): Developed in Collaboration With the American Society of Echocardiography, American Society of Nuclear Cardiology, Heart Rhythm Society, Society of Cardiovascular Anesthesiologists, Society for Cardiovascular Angiography and Interventions, Society for Vascular Medicine and Biology, and Society for Vascular Surgery. Circulation 2007; 116: 1971-96.

19. Kaplan NM. Beta-blockers in hypertension: adding insult to injury. J Am Coll Cardiol 2008; 52: 1490-1. 
20. Williams B, Lacy PS, CAFE and the ASCOT (Anglo-Scandinavian Cardiac Outcomes Trial) Investigators. Impact of heart rate on central aortic pressures and hemodynamics: analysis from the CAFE (Conduit Artery Function Evaluation) Study: CAFE-Heart Rate. J Am Coll Cardiol 2009; 54: 705-13.

21. London MJ. Perioperative beta-blockade, discontinuation, and complications: do you really know it when you see it? Anesthesiology 2009; 111: 690-4.

22. Beattie WS, Wijeysundera DN, Karkouti K, et al. Acute surgical anemia influences the cardioprotective effects of $\beta$ blockade: a single-center, propensity matched cohort study. Anesthesiology 2010; 112: 25-33.

23. van Klei WA, Bryson GL, Yang H, Forster AJ. Effect of betablocker prescription on the incidence of postoperative myocardial infarction after hip and knee arthroplasty. Anesthesiology 2009; 111: 717-24.

24. Comfere T, Sprung J, Kumar MM, et al. Angiotensin system inhibitors in a general surgical population. Anesth Analg 2005; 100: 636-44.

25. Miceli A, Capoun R, Fino $C$, et al. Effects of angiotensin-converting enzyme inhibitor therapy on clinical outcome in patients undergoing coronary artery bypass grafting. J Am Coll Cardiol 2009; 54: 1778-84.

26. Tricoci P, Allen JM, Kramer JM, Califf RM, Smith SC Jr. Scientific evidence underlying the ACC/AHA clinical practice guidelines. JAMA 2009; 301: 831-41.

27. Shaneyfelt TM, Centor RM. Reassessment of clinical practice guidelines: go gently into that good night. JAMA 2009; 301: 868-9.

28. Lee TH, Marcantonio ER, Mangione CM, et al. Derivation and prospective validation of a simple index for prediction of cardiac risk of major noncardiac surgery. Circulation 1999; 100: 1043-9.

29. Boersma E, Kertai MD, Schouten $O$, et al. Perioperative cardiovascular mortality in noncardiac surgery: validation of the Lee cardiac risk index. Am J Med 2005; 118: 1134-41.

30. Schouten $O$, Kok NF, Hoedt MT, van Laanen JH, Poldermans D. The influence of aneurysm size on perioperative cardiac outcome in elective open infrarenal aortic aneurysm repair. J Vasc Surg 2006; 44: 435-41.

31. Cuthbertson BH, Amiri AR, Croal BL, et al. Utility of B-type natriuretic peptide in predicting perioperative cardiac events in patients undergoing major non-cardiac surgery. $\mathrm{Br} \mathrm{J}$ Anaesth 2007; 99: 170-6.

32. Hoeks SE, op Reimer WJ, van Gestel YR, et al. Preoperative cardiac risk index predicts long-term mortality and health status. Am J Med 2009; 122: 559-65.

33. Karthikeyan $G$, Moncur RA, Levine $O$, et al. Is a pre-operative brain natriuretic peptide or $\mathrm{N}$-terminal pro-B-type natriuretic peptide measurement an independent predictor of adverse cardiovascular outcomes within 30 days of noncardiac surgery? A systematic review and meta-analysis of observational studies. J Am Coll Cardiol 2009; 54: 1599-606.

34. Mangano DT, Layug EL, Wallace A, Tateo I. Effect of atenolol on mortality and cardiovascular morbidity after noncardiac surgery. Multicenter Study of Perioperative Ischemia Research Group. N Engl J Med 1996; 335: 1713-20.

35. Weiskopf RB, Feiner J, Hopf H, et al. Heart rate increases linearly in response to acute isovolemic anemia. Transfusion 2003; 43: 235-40.

36. Bangalore S, Sawhney S, Messerli FH. Relation of beta-blockerinduced heart rate lowering and cardioprotection in hypertension. J Am Coll Cardiol 2008; 52: 1482-9.

37. Cucherat M. Quantitative relationship between resting heart rate reduction and magnitude of clinical benefits in post-myocardial infarction: a meta-regression of randomized clinical trials. Eur Heart J 2007; 28: 3012-9.
38. Fox K, Ford I, Steg $P G$, et al. Heart rate as a prognostic risk factor in patients with coronary artery disease and left-ventricular systolic dysfunction (BEAUTIFUL): a subgroup analysis of a randomised controlled trial. Lancet 2008; 372: 817-21.

39. Kolloch $R$, Legler UF, Champion A, et al. Impact of resting heart rate on outcomes in hypertensive patients with coronary artery disease: findings from the INternational VErapamil-SR/trandolapril STudy (INVEST). Eur Heart J 2008; 29: 1327-34.

40. Biccard BM, Sear JW, Foex P. Meta-analysis of the effect of heart rate achieved by perioperative beta-adrenergic blockade on cardiovascular outcomes. Br J Anaesth 2008; 100: 23-8.

41. Beattie WS, Wijeysundera DN, Karkouti K, McCluskey S, Tait G. Does tight heart rate control improve beta-blocker efficacy? An updated analysis of the noncardiac surgical randomized trials. Anesth Analg 2008; 106: 1039-48.

42. Bangalore S, Wetterslev J, Pranesh S, Sawhney S, Gluud C, Messerli FH. Perioperative beta blockers in patients having noncardiac surgery: a meta-analysis. Lancet 2008; 372: 1962-76.

43. Marwick TH, Branagan H, Venkatesh B, Stewart S, STRATIFY Investigators. Use of a nurse-led intervention to optimize betablockade for reducing cardiac events after major noncardiac surgery. Am Heart J 2009; 157: 784-90.

44. Joannides $R$, Moore $N$, Iacob $M$, et al. Comparative effects of ivabradine, a selective heart rate-lowering agent, and propranolol on systemic and cardiac haemodynamics at rest and during exercise. Br J Clin Pharmacol 2006; 61: 127-37.

45. Fox K, Ford I, Steg PG, Tendera M, Ferrari R, BEAUTIFUL Investigators. Ivabradine for patients with stable coronary artery disease and left-ventricular systolic dysfunction (BEAUTIFUL): a randomised, double-blind, placebo-controlled trial. Lancet 2008; 372: 807-16.

46. Eggers KM, Lind L, Ahlstrom H, et al. Prevalence and pathophysiological mechanisms of elevated cardiac troponin I levels in a population-based sample of elderly subjects. Eur Heart J 2008; 29: 2252-8.

47. Omland T, de Lemos JA, Sabatine MS, et al. A sensitive cardiac troponin $\mathrm{T}$ assay in stable coronary artery disease. N Engl J Med 2009; DOI: 10.1056/NEJMoa0805299.

48. Lowenstein E. Perianesthetic ischemic episodes cause myocardial infarction in human-a hypothesis confirmed. Anesthesiology 1985; 62: 103-6.

49. Mangano DT, Browner WS, Hollenberg M, London MJ, Tubau $J F$, Tateo IM. Association of perioperative myocardial ischemia with cardiac morbidity and mortality in men undergoing noncardiac surgery. The Study of Perioperative Ischemia Research Group. N Engl J Med 1990; 323: 1781-8.

50. Erne P, Schoenenberger AW, Burckhardt D, et al. Effects of percutaneous coronary interventions in silent ischemia after myocardial infarction: the SWISSI II randomized controlled trial. JAMA 2007; 297: 1985-91.

51. Scirica BM, Morrow DA, Budaj A, et al. Ischemia detected on continuous electrocardiography after acute coronary syndrome: observations from the MERLIN-TIMI 36 (Metabolic Efficiency With Ranolazine for Less Ischemia in Non-ST-Elevation Acute Coronary Syndrome-Thrombolysis In Myocardial Infarction 36) trial. J Am Coll Cardiol 2009; 53: 1411-21.

52. Tzivoni D, Krucoff $M W$. Continuous ST-segment monitoring in contemporary acute coronary syndrome patients: the magic of MERLIN-TIMI 36. J Am Coll Cardiol 2009; 53: 1422-4.

53. Hare GM, Tsui AK, McLaren AT, Ragoonanan TE, Yu J, Mazer $C D$. Anemia and cerebral outcomes: many questions, fewer answers. Anesth Analg 2008; 107: 1356-70.

54. Beattie WS, Karkouti K, Wijeysundera DN, Tait G. Risk associated with preoperative anemia in noncardiac surgery: a singlecenter cohort study. Anesthesiology 2009; 110: 574-81. 
55. Ragoonanan TE, Beattie WS, Mazer CD, et al. Metoprolol reduces cerebral tissue oxygen tension after acute hemodilution in rats. Anesthesiology 2009; 111: 988-1000.

56. Dagnino J, Prys-Roberts C. Assessment of beta-adrenoceptor blockade during anesthesia in humans: use of isoproterenol doseresponse curves. Anesth Analg 1985; 64: 305-11.

57. Zaugg M, Bestmann L, Wacker J, et al. Adrenergic receptor genotype but not perioperative bisoprolol therapy may determine cardiovascular outcome in at-risk patients undergoing surgery with spinal block: the Swiss Beta Blocker in Spinal Anesthesia (BBSA) study: a double-blinded, placebo-controlled, multicenter trial with 1-year follow-up. Anesthesiology 2007; 107: 33-44.

58. Kurnik D, Li C, Sofowora GG, et al. Beta-1-adrenoceptor genetic variants and ethnicity independently affect response to betablockade. Pharmacogenet Genomics 2008; 18: 895-902.

59. Joyner MJ. Not so fast: intrinsic heart rate vs. beta-adrenergic responsiveness in the aging human heart. J Appl Physiol 2008; 105: 3-4.
60. Dunkelgrun M, Hoeks SE, Welten GM, et al. Anemia as an independent predictor of perioperative and long-term cardiovascular outcome in patients scheduled for elective vascular surgery. Am J Cardiol 2008; 101: 1196-200.

61. Kindermann M, Maack $C$, Schaller $S$, et al. Carvedilol but not metoprolol reduces beta-adrenergic responsiveness after complete elimination from plasma in vivo. Circulation 2004; 109: 3182-90.

62. Stoschitzky K, Stoschitzky G, Brussee H, Bonelli C, Dobnig H. Comparing beta-blocking effects of bisoprolol, carvedilol and nebivolol. Cardiology 2006; 106: 199-206.

63. Fu GS, Huang H, Chen F, et al. Carvedilol ameliorates endothelial dysfunction in streptozotocin-induced diabetic rats. Eur J Pharmacol 2007; 567: 223-30.

64. Phillips RA, Fonseca V, Katholi RE, et al. Demographic analyses of the effects of carvedilol vs metoprolol on glycemic control and insulin sensitivity in patients with type 2 diabetes and hypertension in the Glycemic Effects in Diabetes Mellitus: CarvedilolMetoprolol Comparison in Hypertensives (GEMINI) study. J Cardiometab Syndr 2008; 3: 211-7. 